\title{
Outcome of Pulmonary Spherical Ground-glass Opacities on Ct in Patients With Coronavirus Disease 2019 (Covid-19): A Retrospective Analysis
}

\section{Wangjia Li}

The first affiliated hospital of Chongqing medical university

\section{Liangbo Hu}

Yongchuan Hospital of Chongqing Medical University

Junhao Huang

Yongchuan Hospital of Chongqing Medical University

\section{Fajin Lv}

The first affiliated hospital of chongqing medical university

\section{Binjie Fu}

The first affiliated hospital of chongqing medical university

\section{Zhigang Chu ( $\square$ chuzg0815@163.com)}

Chongqing Medical University First Affiliated Hospital https://orcid.org/0000-0002-2441-8132

\section{Research article}

Keywords: Coronavirus, Pneumonia, COVID-19, Computed tomography, Ground glass nodules

Posted Date: May 28th, 2020

DOI: https://doi.org/10.21203/rs.3.rs-30665/v1

License: () (1) This work is licensed under a Creative Commons Attribution 4.0 International License. Read Full License 


\section{Abstract}

Background: Pulmonary spherical ground-glass opacities (GGOs) are commonly detected on initial chest CT scan in patients with coronavirus disease 2019 (COVID-19).We aimed to investigate the evolution of spherical GGOs to better understand their clinical significance.

Materials and Methods:A retrospective study of 33 consecutive patients with confirmed COVID-19 and pulmonary spherical GGOs was performed from January 21, 2020, to March 6, 2020. The initial and follow-up CT images and clinical data were reviewed. The initial CT manifestations of spherical GGOs and their subsequent changes were mainly evaluated.

Results:A total of 101 pulmonary spherical GGOs, including 38 with and 63 without consolidation, were found in 33 patients. Of the 101 spherical GGOs, 71 (70.3\%) and 30 (29.7\%) showed progression and direct absorption on follow-up CT images, respectively. GGOs with consolidation were more likely to progress than those without (84.2\% vs. $61.9 \%, p=0.017)$. The 71 progressed lesions mainly showed an increase in size and/or density and most (70.4\%) of them extended toward the pleura and developed from spherical to patchy. Internal consolidation appeared and increased in 18 (25.4\%) and 22 (31.0\%) lesions, respectively. During absorption, all the previous progressed and directly absorbed lesions exhibited a simultaneous decrease in size and density. On each patient's final CT, more lesions with progression had a residual mixed GGO (40.8\% vs. $6.7 \%, p=0.002)$ and fewer had pure GGO $(39.4 \%$ vs. $60.0 \%, p=0.016)$ than those with direct absorption.

Conclusion: In patients with COVID-19, most pulmonary spherical ground-glass opacities would progress, especially those with consolidation, and develop into patchy, subpleural lesions.

\section{Introduction}

The current outbreak of coronavirus disease 2019 (COVID-19) was first reported in Wuhan, China, on December 31, 2019, and it subsequently spread worldwide [1]. With an increasing number of confirmed cases, the World Health Organization declared COVID-19 a global health emergency. Although real-time reverse transcription polymerase chain reaction (RT-PCR) remains the reference standard for diagnosing COVID-19 [2], the emergence of false negatives $[3,4]$ and the requirement for significant professional skills restricted prompt diagnosis of suspected cases. Some investigations in the primary epidemic area reported that chest CT had greater sensitivity for COVID19 diagnosis compared with initial RT-PCR $[5,6]$, making chest CT an important tool in the early detection, diagnosis, and evaluation of the disease course [7].

To date, there have been several studies on imaging of COVID-19 [8-11]. Pan et al. [8] and Chung et al. [9] investigated the initial and follow-up CT findings of 63 and 21 confirmed patients, respectively, and found that the CT manifestations of lesions were various and changed rapidly. Pan et al. [10] studied the temporal CT changes of COVID-19 from initial diagnosis until recovery and identified four stages of evolution on chest CT. They also found that spherical ground-glass opacities (GGOs) were the initial CT manifestation, which was consistent with the result of Chung et al. [8]. However, no research has focused on the development of spherical GGOs and their changes following treatment.

Because extensive, patchy GGOs and GGOs with consolidation were common at advanced stage of COVID-19, we hypothesized that some spherical GGOs were the precursors of patchy GGOs with or without consolidation. If this hypothesis is confirmed, it can aid in the earlier diagnosis of suspected cases. This study investigated the outcome 
of spherical GGOs on CT to reveal their evolution and relationship with patchy lesions. The results will allow radiologists and clinicians to better understand the evolution of spherical GGOs on chest CT, hopefully diagnose the disease earlier, and identify patients with a worse prognosis.

\section{Materials And Methods}

This retrospective study used non-identifiable patient data for analysis and was approved by the institutional review board. Therefore, the requirement for informed consent was waived.

\section{Study Population}

From January 21, 2020, to March 6, 2020, patients with confirmed COVID-19 were retrospectively reviewed. Inclusion criteria: (1) presence of at least two follow-up CT scans, (2) the patients' clinical data were complete. Exclusion criteria: (1) patients without pulmonary lesions, (2) patients without lesions presenting as spherical GGOs on initial chest CT, and (3) the interval between initial and follow-up CTs was $>7$ days.

\section{Ct Examinations}

All patients underwent a non-contrast CT upon admission to the hospital with follow-up CT scans at $<7$ days. Chest CT scans were performed using two MDCT scanners (Philips Brilliance iCT and Siemens SOMATOM go.Top) with the following parameters: tube voltage, $120 \mathrm{kV}$; automatic tube current modulation; beam pitch, 0.758/1.5; detector collimation, $0.625 / 0.6 \mathrm{~mm}$; rotation time, $0.5 \mathrm{~s}$; matrix size, $512 \times 512$; and section thickness and interval, 5.0 and $5.0 \mathrm{~mm}$, respectively. All patients were in a supine position and scanned from the thoracic inlet to the lung base at the end of inspiration. CT images were reconstructed using a medium sharp reconstruction algorithm with a section thickness of $1 \mathrm{~mm}$.

\section{Analysis Of Ct Characteristics}

Image analysis was evaluated on a PACS workstation (Vue PACS, Carestream Health, Inc) by two experienced radiologists (Z.G.C and F.J.L with 10 and 20 years of experience in thoracic radiology, respectively). They were blinded to clinical data and independently reviewed the images on both lung (width, $1500 \mathrm{HU}$; level, $-500 \mathrm{HU}$ ) and mediastinal (width, $350 \mathrm{HU}$; level, $40 \mathrm{HU}$ ) settings. Discrepancies were resolved by consensus.

For each spherical GGO, the initial CT scan was evaluated for the following characteristics: (a) lesion location, (b) relationship with the pleura (close to pleura, under the pleura, distant from the pleura), (c) lesion size, (d) CT value, (e) consolidation (yes or no), (f) uniformity of density (homogenous or heterogeneous), and (g) lesion border (illdefined or well-defined). The relationship between the lesion and the pleura was considered as under the pleura if the distance between lesion and pleura was $\leq 2 \mathrm{~cm}$; if $>2 \mathrm{~cm}$, it was considered as distant from the pleura. All patients underwent follow-up chest CT scans, and the following results were reviewed: (a) the size and density changes of previous spherical GGOs, (b) changes of lesion border, (c) changes of consolidation, (d) the appearance of air bronchogram, and (f) the manifestations of residual lesions on the latest CT scan. Based on the changes of initial lesions on follow-up CT scans, spherical GGOs were divided into two groups: those that showed progression and those that were directly absorbed. 


\section{Clinical Features}

Clinical and laboratory data of the patients were collected by one radiologist (W.J.L). Clinical data, including age, gender, clinical type, initial symptoms, length of hospitalization, numbers of scans, and the interval between the adjacent scans were recorded. Laboratory findings such as white blood cell count, neutrophil count and percentage, lymphocyte count and percentage, C-reactive protein, erythrocyte sedimentation rate, and lactate dehydrogenase, were also recorded. These laboratory tests were performed upon admission to the hospital.

\section{Statistical Analysis}

All data were analyzed using SPSS 20.0 (SPSS, Chicago, III). Data were expressed as mean \pm standard deviation for continuous variables and as numbers and percentages for categorical variables. The continuous variables used the analysis of Variance or Wilcoxon rank-sum test, and categorical variables were analyzed using the Pearson $\chi 2$ test or Fisher exact test. A p value of $<0.05$ was considered significant.

\section{Results}

\section{Clinical and Laboratory Characteristics}

A total of 33 patients were included in this study (Fig. 1), including 25 men (mean age, $44 \pm 15$ years; range, 16-73) and 8 women (mean age, $51 \pm 13$ years; range, 40-67). Of the 33 patients, $29(87.9 \%)$ had a history of long-term residence in Wuhan or exposure to infected patients. The clinical and laboratory characteristics of the patients with COVID-19 are listed in Table 1. The most common symptoms were fever $(26 / 33,78.8 \%)$ and cough $(24 / 33,72.7 \%)$. Most patients had a normal white blood cell count $(26 / 33,78.8 \%)$ and neutrophil count $(29 / 33,87.9 \%)$. The lymphocyte count was either low $(17 / 33,51.5 \%)$ or normal $(16 / 33,48.5 \%)$. C-reactive protein was elevated in 19/33 cases (57.6\%). Of the 33 cases, $24(72.7 \%)$ and 31 (93.9\%) showed elevated erythrocyte sedimentation rates and lactate dehydrogenase levels, respectively. 
Table 1

Clinical and laboratory characteristics of patients with COVID-19

\begin{tabular}{|c|c|}
\hline Characteristics & All patients $(n=33)$ \\
\hline Age (y) & $46 \pm 15(16-73)$ \\
\hline \multicolumn{2}{|l|}{ Gender } \\
\hline Male & $25(75.8)$ \\
\hline Female & $8(24.2)$ \\
\hline \multicolumn{2}{|l|}{ Clinical type } \\
\hline Mild & $1(3.0)$ \\
\hline Moderate & $29(87.9)$ \\
\hline Severe & $3(9.1)$ \\
\hline Critical & $0(0)$ \\
\hline \multicolumn{2}{|l|}{ Initial symptoms } \\
\hline Cough & $24(72.7)$ \\
\hline Expectoration & $6(18.2)$ \\
\hline Fever & $26(78.8)$ \\
\hline Low grade fever $\left(37.5-38.0^{\circ} \mathrm{C}\right)$ & $13(39.4)$ \\
\hline Moderate grade fever $\left(38.1-39.0^{\circ} \mathrm{C}\right)$ & $10(30.3)$ \\
\hline High grade fever $\left(>39.1^{\circ} \mathrm{C}\right)$ & $0(0)$ \\
\hline Myalgia or fatigue & $9(27.3)$ \\
\hline \multicolumn{2}{|l|}{ Laboratory findings } \\
\hline White blood cell count $\left(\times 10^{9} / \mathrm{L}\right)$ & $4.6 \pm 1.5(1.5-7.8)$ \\
\hline Neutrophil count $\left(\times 10^{9} / \mathrm{L}\right)$ & $3.2 \pm 1.4(0.8-6.4)$ \\
\hline Neutrophil percentage (\%) & $64 \pm 11(38-96)$ \\
\hline Lymphocyte count $\left(\times 10^{9} / \mathrm{L}\right)$ & $1.1 \pm 0.5(0.4-2.8)$ \\
\hline Lymphocyte percentage (\%) & $25 \pm 9(11-49)$ \\
\hline C-reactive protein (mg/L) & $23.4 \pm 27.1(0.5-94.9)$ \\
\hline Erythrocyte sedimentation rate $(\mathrm{mm} / \mathrm{h})$ & $42 \pm 31(2-98)$ \\
\hline Lactate dehydrogenase (U/L) & $534 \pm 171(214-1024)$ \\
\hline The length of hospitalization (d) & $14 \pm 4(7-20)$ \\
\hline Number of scans & $4 \pm 1(3-7)$ \\
\hline The interval between the adjacent scans (d) & $5 \pm 3(1-10)$ \\
\hline
\end{tabular}




\section{Characteristics}

All patients $(n=33)$

Data are expressed as $\mathrm{n}(\%)$ or mean \pm SD (range)

Abbreviations: COVID-19, coronavirus disease 2019.

\section{Initial Ct Findings Of Spherical Ggos}

The mean duration from illness onset to initial CT examination was 3 days. Initial chest CT findings of 101 spherical GGOs are presented in Table 2. There were 65/101 (64.4\%) lesions in the lower lobes and 89/101 (88.1\%) lesions in the peripheral zone. The mean size and CT value of lesions were $16.6 \pm 8.0 \mathrm{~mm}$ and $-390 \pm 182 \mathrm{HU}$, respectively. Of the 101 lesions, 38 (37.6\%) contained internal consolidation, $75(74.3 \%)$ had heterogeneous density, and 65 (64.4\%) had an ill-defined border. 
Table 2

Initial chest CT findings of 101 spherical GGOs in 33 patients with COVID-19.

\begin{tabular}{|c|c|c|c|}
\hline & $\begin{array}{l}\text { Progressed GGOs } \\
(n=71)\end{array}$ & $\begin{array}{l}\text { Absorbed GGOs } \\
(n=30)\end{array}$ & $P$-value \\
\hline \multicolumn{4}{|l|}{ Location } \\
\hline Right upper lobe & $9(12.7)$ & $6(20.0)$ & \multirow[t]{5}{*}{$0.317^{\star \star}$} \\
\hline Right middle lobe & $4(5.6)$ & $2(6.7)$ & \\
\hline Right lower lobe & $29(40.8)$ & $10(33.3)$ & \\
\hline Left upper lobe & $8(11.3)$ & 7 (23.3) & \\
\hline Left lower lobe & $21(29.6)$ & $5(16.7)$ & \\
\hline \multicolumn{4}{|c|}{ The relationship with pleura } \\
\hline Close to pleura & $27(38.0)$ & $9(30.0)$ & \multirow[t]{3}{*}{$0.616^{*}$} \\
\hline Under the pleura & $35(49.3)$ & $18(60.0)$ & \\
\hline Distant from pleura & $9(12.7)$ & $3(10.0)$ & \\
\hline \multirow[t]{2}{*}{ Lesion size (mm) } & $15.9 \pm 7.5$ & $18.4 \pm 9.1$ & \multirow[t]{2}{*}{$0.100^{\&}$} \\
\hline & $(6.5-47.3)$ & $(8.65-53.5)$ & \\
\hline \multirow[t]{2}{*}{ CT value(HU) } & $-383 \pm 195$ & $-410 \pm 152$ & \multirow[t]{2}{*}{$0.397^{\&}$} \\
\hline & $(-78--768)$ & $(-94--630)$ & \\
\hline \multicolumn{4}{|l|}{ Consolidation } \\
\hline Yes & $32(45.1)$ & $6(20.0)$ & \multirow[t]{2}{*}{$0.017^{\star}$} \\
\hline No & 39 (54.9) & $24(80.0)$ & \\
\hline \multicolumn{4}{|l|}{ Uniformity of density } \\
\hline Homogeneous & $20(28.2)$ & $6(20.0)$ & \multirow[t]{2}{*}{$0.391^{*}$} \\
\hline Heterogeneous & $51(71.8)$ & $24(80.0)$ & \\
\hline \multicolumn{4}{|l|}{ Border } \\
\hline III-defined & $45(63.4)$ & $20(66.6)$ & \multirow[t]{2}{*}{$0.297^{*}$} \\
\hline Well-defined & $26(36.6)$ & 10 (33.3) & \\
\hline \multicolumn{4}{|c|}{ Data are expressed as $n(\%)$ or mean \pm SD (range) } \\
\hline \multicolumn{4}{|l|}{${ }^{\star \star}$ Fisher exact test. } \\
\hline \multicolumn{4}{|c|}{ \& Wilcoxon rank sum test } \\
\hline \multicolumn{4}{|c|}{ Abbreviations: COVID-19, coronavirus disease 2019; GGOs, ground-glass opacities. } \\
\hline
\end{tabular}


Based on the changes of the 101 lesions during follow-up, 71 (70.3\%) progressed and 30 (29.7\%) directly absorbed. Initial spherical GGOs with consolidation were more likely to progress than those without consolidation (84.2\% vs. $61.9 \%, p=0.017)$.

\section{Follow-up Ct Scans}

There was a total of 91 (mean, $3 \pm 1$; range, 2-6) follow-up CT scans with a mean interval of $5 \pm 1$ days (range, 110 days). The CT manifestations and changes of spherical GGOs during progressing to the peak or direct absorption are presented in Table 3. As progressing to the peak, the lesions usually showed a simultaneous increase in size and density (84.5\%), a well-defined border (81.7\%), and newly emerging consolidation (25.4\%) or increased consolidation (31.0\%) (Fig. 2A, B). Regarding the shape of 71 progressed spherical GGOs, 50 (70.4\%) developed from spherical into patchy (fan-shaped, 23/71 (32.4\%); irregular, 20/71 (28.2\%); crescent-shaped, 4/71 (5.6\%); and triangular, 3/71 (4.2\%)), while 21/71 (29.6\%) remained unchanged. Their extent usually moved toward the pleura and further expanded along the subpleural zone (Fig. 2B). During absorption, the directly absorbed lesions maintained their original shape and demonstrated a simultaneous decrease in size and density $(26 / 30$, $86.7 \%$ ) (Fig. 3A, B, C, D). They usually had an ill-defined border (27/30, 90.0\%) and no newly emerging consolidation $(24 / 30,80.0 \%)$ or disappearance $(5 / 30,16.7 \%)$ of previous consolidation. 
Table 3

The CT manifestations and changes of spherical GGOs during progressing to the peak or absorption

\begin{tabular}{|c|c|c|}
\hline & $\begin{array}{l}\text { Progressed lesions } \\
(n=71)\end{array}$ & $\begin{array}{l}\text { Absorbed lesions } \\
(n=30)\end{array}$ \\
\hline \multicolumn{3}{|l|}{ Changes of size and density } \\
\hline Increased size and increased density & $60(84.5)$ & / \\
\hline Only increased size & $6(8.5)$ & / \\
\hline Only increased size & $5(7.0)$ & / \\
\hline Decreased lesion size and decreased density & / & $26(86.7)$ \\
\hline Only decreased size & / & $2(6.7)$ \\
\hline Only decreased size & / & $2(6.7)$ \\
\hline \multicolumn{3}{|l|}{ Changes of lesion border } \\
\hline Well-defined to ill-defined & $3(4.2)$ & $10(33.3)$ \\
\hline III-defined to well-defined & $35(49.3)$ & $0(0)$ \\
\hline Remain well-defined & $23(32.4)$ & $3(10.0)$ \\
\hline Remain ill-defined & $10(14.1)$ & $17(56.7)$ \\
\hline \multicolumn{3}{|l|}{ Changes of consolidation } \\
\hline Appearance or increase & $40(56.3)$ & $1(3.3)$ \\
\hline Disappearance & $5(7.0)$ & $5(16.7)$ \\
\hline Without consolidation & $26(3.6)$ & $24(80.0)$ \\
\hline \multicolumn{3}{|l|}{ Appearance of air bronchogram } \\
\hline Yes & $14(20)$ & $1(3.3)$ \\
\hline No & $57(80)$ & $29(96.7)$ \\
\hline \multicolumn{3}{|l|}{ CT manifestations on the final CT scan } \\
\hline Totally disappeared & $14(19.7)$ & $10(33.3)$ \\
\hline Pure GGOs & $28(39.4)$ & $18(60.0)$ \\
\hline Mixed GGOs & $29(40.8)$ & $2(6.7)$ \\
\hline \multicolumn{3}{|l|}{ Data are expressed as $\mathrm{n}(\%)$} \\
\hline \multicolumn{3}{|c|}{ Mixed GGO indicates GGO with fibrous stripes and/or consolidation } \\
\hline Abbreviations: GGOs, ground-glass opacities. & & \\
\hline
\end{tabular}

The mean follow-up time for all lesions was $20 \pm 6$ days (range, 12-32 days). The mean follow-up time for lesions with progression ( $21 \pm 6$ days; range, $12-32$ days) and those with direct absorption (18 \pm 6 days; range, 14-27 
days) was similar $(p=0.199)$. During absorption, the previous progressed lesions mainly exhibited a simultaneous decrease in size and density (Fig. 2C, D). On the final CT, 14 (19.7\%) progressed lesions and 10 (33.3\%) directly absorbed lesions had completely disappeared. More progressed lesions had residual mixed GGOs (40.8\% vs. $2 / 30$ $6.7 \%, p=0.002)$, but fewer had pure GGOs $(39.4 \%$ vs. $60.0 \%, P=0.016)$ than the directly absorbed lesions.

Based on the initial CT manifestations of all pulmonary lesions, patients were divided into three groups: those with only spherical GGOs (group 1), those with spherical GGOs and a few patchy lesions (group 2), and those with patchy lesions and a few spherical GGOs (group 3). The absorption of spherical GGOs in different groups on the final CT is presented in Table 4. Compared with groups 1 and 2, more spherical GGOs in group 3 progressed ( $p=$ $0.000)$. The absorption of lesions in the different groups differed significantly $(p=0.002)$. Compared with lesions in groups 1 and 2, those in group 3 had more residual GGOs $(p=0.000)$, especially the mixed GGOs $(p=0.007)$.

Table 4

The absorption of spherical GGOs in different groups on the latest followup CT

\begin{tabular}{|lccc|}
\hline & $\begin{array}{c}\text { Group 1 } \\
(\mathbf{n = 1 0})\end{array}$ & $\begin{array}{c}\text { Group 2 } \\
(\mathbf{n}=\mathbf{5 8})\end{array}$ & $\begin{array}{c}\text { Group 3 } \\
(\mathbf{n}=\mathbf{3 3})\end{array}$ \\
\hline Progressed lesions & $6(60.0)$ & $36(62.1)$ & $30(90.9)$ \\
\hline Absorbed lesions & $4(40.0)$ & $22(37.9)$ & $3(9.1)$ \\
\hline Manifestations on the final CT scan & & & \\
\hline Totally disappeared & $6(60.0)$ & $17(29.3)$ & $1(3.0)$ \\
\hline Pure GGOs & $3(30.0)$ & $26(44.8)$ & $17(51.5)$ \\
\hline Mixed GGOs & $1(10.0)$ & $15(25.9)$ & $15(45.4)$ \\
\hline Mixed GGO indicates GGO with fibrous stripes and/or consolidation \\
Data are expressed as n (\%) \\
Abbreviations: GGOs, ground-glass opacities. & & \\
\hline
\end{tabular}

\section{Discussion}

The chest CT manifestations of coronavirus disease 2019 (COVID-19) pneumonia are various, including groundglass opacities (GGOs), fibrous stripes, and consolidation [12]. Previous studies investigating the clinical characteristics and CT changes of COVID-19 pneumonia had confirmed that spherical GGOs were one of the initial, common radiological manifestations $[8,9,13-15]$. However, there were some cases with only patchy lesions on the initial scan; therefore, the clinical significance of spherical GGOs was unclear. Prior to understanding the significance of spherical GGOs, several questions must be answered. First, whether there is relationship between spherical and patchy GGOs. Second, how do spherical GGOs change following treatment. Third, whether there are differences in the outcomes of spherical GGOs with different initial manifestations. Last, whether the short-term follow-up CT manifestations of spherical GGOs are identical or not. In this study, all these issues were studied.

In the present study, most spherical GGOs were faint, heterogeneous, and ill-defined, with a subpleural distribution on initial CT scan. During follow-up, most showed progression in size and/or density. During progression, their 
borders became more well-defined and internal consolidation either appeared or increased. On subsequent axial CT imaging, most of the initial spherical GGOs lost their round shape and appeared as patchy lesions. The lesions extended toward the pleura with further expansion along the subpleural zone, indicating that distal alveoli were involved. Therefore, spherical GGOs could be seen as the precursors of patchy ones. This development process might be characteristic for COVID-19 pneumonia, and there should be a significant suspicion for COVID-19 when spherical GGOs showed such changes in an epidemic area.

Since spherical GGOs were early-stage lesions of COVID-19, it was important to know which kind of lesions would progress. We found that spherical GGOs with consolidation were more likely to progress than pure GGOs, suggesting that initial lesions with consolidation are more severe and have a greater possibility of progression. In addition, an increase in lesion size was often accompanied with the appearance or increase of internal consolidation. This indicates exacerbation of the lesion, which is consistent with a previous study [8]. Another study also noted that consolidation indicated disease progression or greater severity for COVID-19 [14]. In addition to internal consolidation, other pulmonary lesions may be related to lesion progression. Compared with patients with no or few patchy GGOs, more spherical lesions in patients with extensive patchy GGOs progressed. By understanding the progression of lung findings over time, it should be possible to identify patients with a greater possibility for disease progression.

During absorption, progressed and directly absorbed spherical GGOs exhibited a simultaneous decrease in size and density. However, the progressed lesions had more residua on the final CT. It is well known that lesion progression can cause more severe lung tissue injury, which results in a slower recovery. Progressed spherical GGOs usually developed into more extensive patchy lesions or had greater consolidation resulting in more severe alveolar and interstitial injury and edema. In contrast, initially pure spherical GGOs appear to be more easily controlled; therefore, most did not develop and had fewer residua.

There were two limitations to this study. First, the current results do not represent the natural development of spherical GGOs because most of the patients received treatment after the initial CT scan. However, we found some important changes in these lesions that can be used for resolving some clinical questions. Second, because some patients with COVID-19 pneumonia were asymptomatic at the early stage, they may have only typical patchy lesions on the initial CT scan. Therefore, it is impossible to confirm whether these patchy lesions originated from spherical ones due to a lack of earlier data. However, it is quite common that spherical GGOs develop into patchy ones, which may aid in the early diagnosis of this disease.

\section{Conclusions}

In conclusion, spherical GGOs are commonly detected in patients with early-stage COVID-19 pneumonia. Following treatment, most of them will progress, especially those with consolidation, and a few will absorb directly. Progressed lesions usually develop into extensive patchy lesions or have more significant consolidation, and absorb more slowly than directly absorbed lesions. These characteristics may be helpful in the early diagnosis of this disease and in identifying patients with a potentially worse prognosis.

\section{Abbreviations}

COVID-19

coronavirus disease 2019; GGOs:ground-glass opacities; RT-PCR:reverse transcriptase polymerase chain reaction. 


\section{Declarations}

\section{Acknowledgments}

Not applicable.

\section{Author information}

1.The First Affiliated Hospital of Chongqing Medical University, 1 \# Youyi Road, Yuanjiagang, Yuzhong district, Chongqing, 400016, People's Republic of China.

Zhi-gang Chu, Wang-jia Li, Fa-jin Lv,Bin-jie Fu.

2.Department of Radiology, Yongchuan Hospital of Chongqing Medical University, Chongqing, China. 402160.

Liangbo Hu, Junhao Huang.

\section{Contributions}

ZGC and WJL contributed to the conceptualization, writing-original draft preparation, writing-review and editing, supervision and visualization. LBH contributed to the writing-review and editing and Supervision. BJF, JHHandFJL contributed to the conceptualization. All authors have approved the final version of the work.

\section{Corresponding authors}

Correspondence toZhigang Chu.

\section{Ethics declarations}

\section{Ethics approval and consent to participate:}

Not applicable.

\section{Consent for publication:}

Not applicable.

\section{Competing interests:}

The authors have no competing interests to disclose.

\section{Funding:}


This study was supported by New Coronavirus Pneumonia Emergency Clinical Research project in Chongqing Medical University, New Coronavirus Pneumonia Emergency project in Science and Technology Bureau of Yongchuan District (No.ycstc,2020nb0204).

\section{References}

1. Velavan TP, Meyer CG. The COVID-19 epidemic. Trop Med Int Health. 2020;25(3):278-80. doi:10.1111/tmi.13383.

2. $10.1038 / \mathrm{d} 41587-020-00010-2$

Sheridan C. Fast, portable tests come online to curb coronavirus pandemic. Nat Biotechnol. 2020 Mar 23. doi: 10.1038/d41587-020-00010-2. [Epub ahead of print].

3. Chan JF, Yuan S, Kok KH, To KK, Chu H, Yang J, et al. A familial cluster of pneumonia associated with the 2019 novel coronavirus indicating person-to-person transmission: a study of a family cluster. Lancet. 2020;395(10223):514-23. doi:10.1016/S0140-6736(20)30154-9.

4. Xie X, Zhong Z, Zhao W, Zheng C, Wang F, Liu J. Chest CT for Typical 2019-nCoV Pneumonia: Relationship to Negative RT-PCR Testing. Radiology. 2020:200343. doi:10.1148/radiol.2020200343. [Epub ahead of print].

5. Ai T, Yang Z, Hou H, Zhan C, Chen C, Lv W, et al. Correlation of Chest CT and RT-PCR Testing in Coronavirus Disease 2019 (COVID-19) in China: A Report of 1014 Cases. Radiology. 2020:200642. doi: 10.1148/radiol. 2020200642. [Epub ahead of print].

6. Fang Y, Zhang H, Xie J, Lin M, Ying L, Pang P, et al Sensitivity of Chest CT for COVID-19: comparison to RTPCR. Radiology. 2020:200432. doi:10.1148/radiol.2020200432. [Epub ahead of print].

7. National Health Commission of the People's Republic of China. The guidlines for the diagnosis and treatment of 2019-nCoV pneumonia (the 5th edition). Available at:

http://www.nhc.gov.cn/yzygj/s7653p/202002/d4b895337e19445f8d728fcaf1e3e13a/files/ab6bec7f93e64e7 f998d802991203cd6.pdf. (Accessed March 8, 2020).

8. Pan Y, Guan H, Zhou S, Wang Y, Li Q, Zhu T, et al. Initial CT findings and temporal changes in patients with the novel coronavirus pneumonia (2019-nCoV): a study of 63 patients in Wuhan, China. Eur Radiol. 2020. doi:10.1007/s00330-020-06731-x. [Epub ahead of print].

9. Chung M, Bernheim A, Mei X, Zhang N, Huang M, Zeng X, et al. CT Imaging Features of 2019 Novel Coronavirus (2019-nCoV). Radiology. 2020;295(1):202-7. doi:10.1148/radiol.2020200230.

10. Pan F, Ye T, Sun P, Gui S, Liang B, Li L, et al. Time Course of Lung Changes On Chest CT During Recovery From 2019 Novel Coronavirus (COVID-19) Pneumonia. Radiology. 2020:200370. doi:10.1148/radiol.2020200370. [Epub ahead of print].

11. Bernheim A, Mei X, Huang M, Yang Y, Fayad ZA, Zhang N, et al. Chest CT Findings in Coronavirus Disease-19 (COVID-19): Relationship to Duration of Infection. Radiology. 2020:200463. doi:10.1148/radiol.2020200463. [Epub ahead of print].

12. Li Y, Xia L. Coronavirus Disease 2019 (COVID-19): Role of Chest CT in Diagnosis and Management. AJR Am J Roentgenol. 2020:1-7. doi:10.2214/AJR.20.22954. [Epub ahead of print].

13. Tian S, Hu W, Niu L, Liu H, Xu H, Xiao SY. Pulmonary Pathology of Early-Phase 2019 Novel Coronavirus (COVID-19) Pneumonia in Two Patients with Lung Cancer. J Thorac Oncol. 2020.

doi:10.1016/j.jtho.2020.02.010. [Epub ahead of print].

Page $13 / 16$ 
14. Song F, Shi N, Shan F, Zhang Z, Shen J, Lu H, et al. Emerging Coronavirus 2019-nCoV Pneumonia. Radiology. 2020;295(1):210-7. doi:10.1148/radiol.2020200274.

15. Zu ZY, Jiang MD, Xu PP, Chen W, Ni QQ, Lu GM, et al. Coronavirus Disease 2019 (COVID-19): A Perspective from China. Radiology. 2020:200490. doi: 10.1148/radiol.2020200490. [Epub ahead of print].

\section{Figures}

Patients with COVID-19 confirmed by RT-PCR between

21 January 2020 and 6 March $2020(n=84)$

Inclusion:

1. presence of at least two follow-up CT scans

2. the patients' clinical data were complete

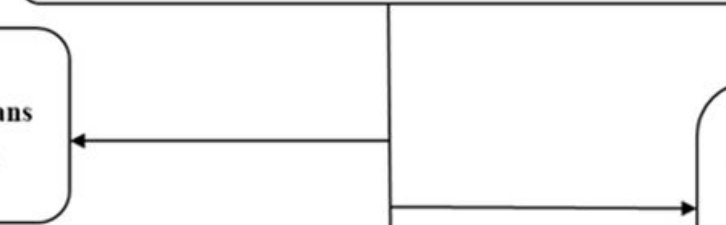

Patients without lesions manifesting as spherica GGO on the initial chest $\mathrm{CT}(\mathrm{n}=32)$

2. Patients without pulmonary lesions $(n=13)$

3. the interval between initial CT and follow-up CT is too long $(>7$ days $)(n=6)$

Thirty-three patients with 101 spherical

GGOs were included.

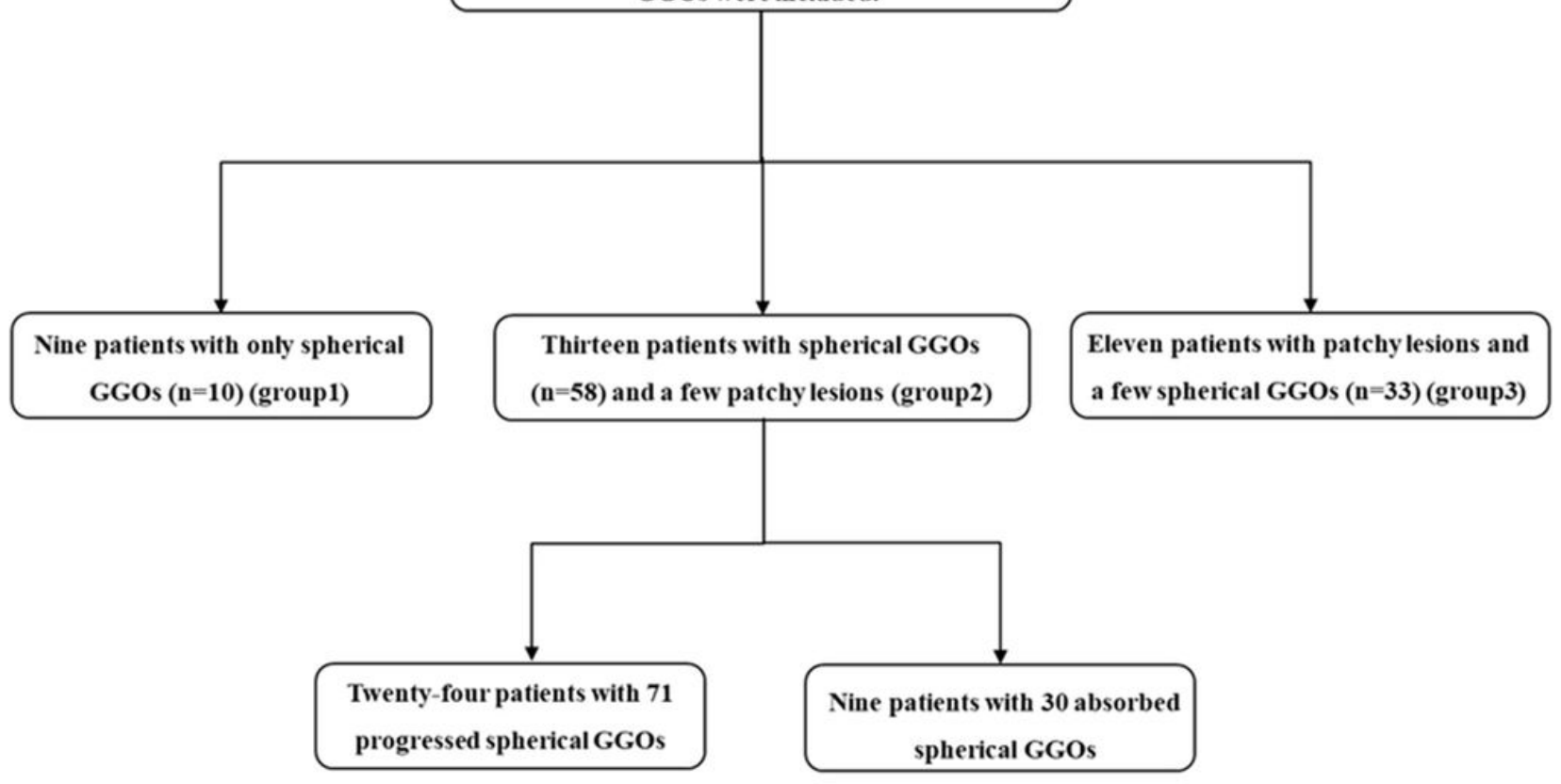

Figure 1

Flowchart of study population 


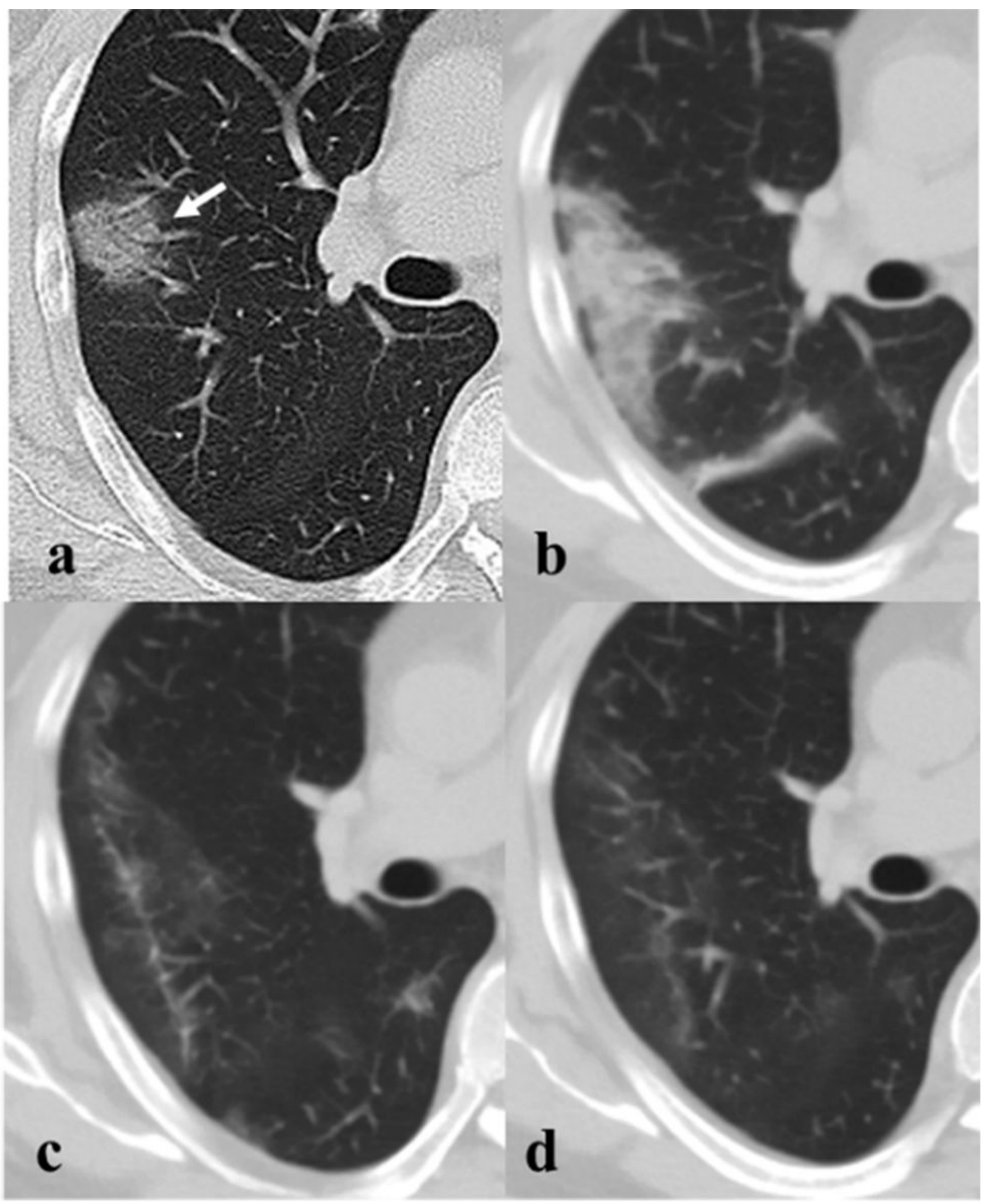

Figure 2

Serial CT images in a 54-year-old man with COVID-19 and progression. Baseline CT image (a) at admission show a subpleural spherical ground-glass opacities (GGO) with consolidation in the right upper lobe. 3 days later, the GGO show a prominent progression, and developed into a patchy consolidation (b). Follow-up CT image (c) on day 8 after admission shows improvement and presented as residual GGO with fibrous stripes. CT scan (d) obtained after 14 days of admission shows near-complete absorption. 


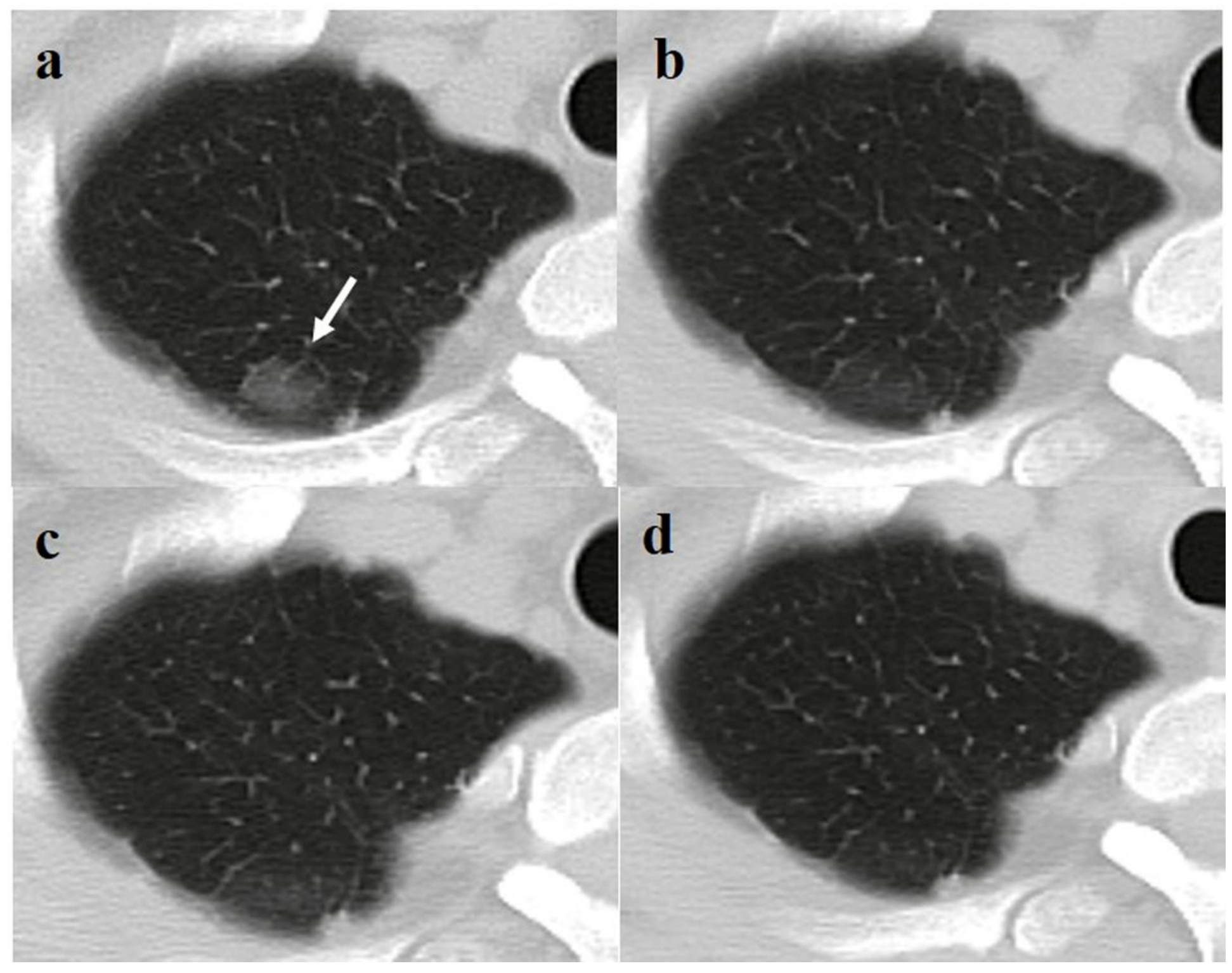

\section{Figure 3}

Serial CT images in a 44-year-old man with COVID-19 and direct absorption. Initial CT scan (a) obtained February 12,2020 shows a right upper lobe subpleural spherical ground-glass opacities (GGO) without consolidation (arrow). Follow-up CT scans (b-d) obtainedfrom February 16, February 19 and February 25, 2020 show the gradual absorption of spherical GGO, and the lesion finally absorbed completely. 